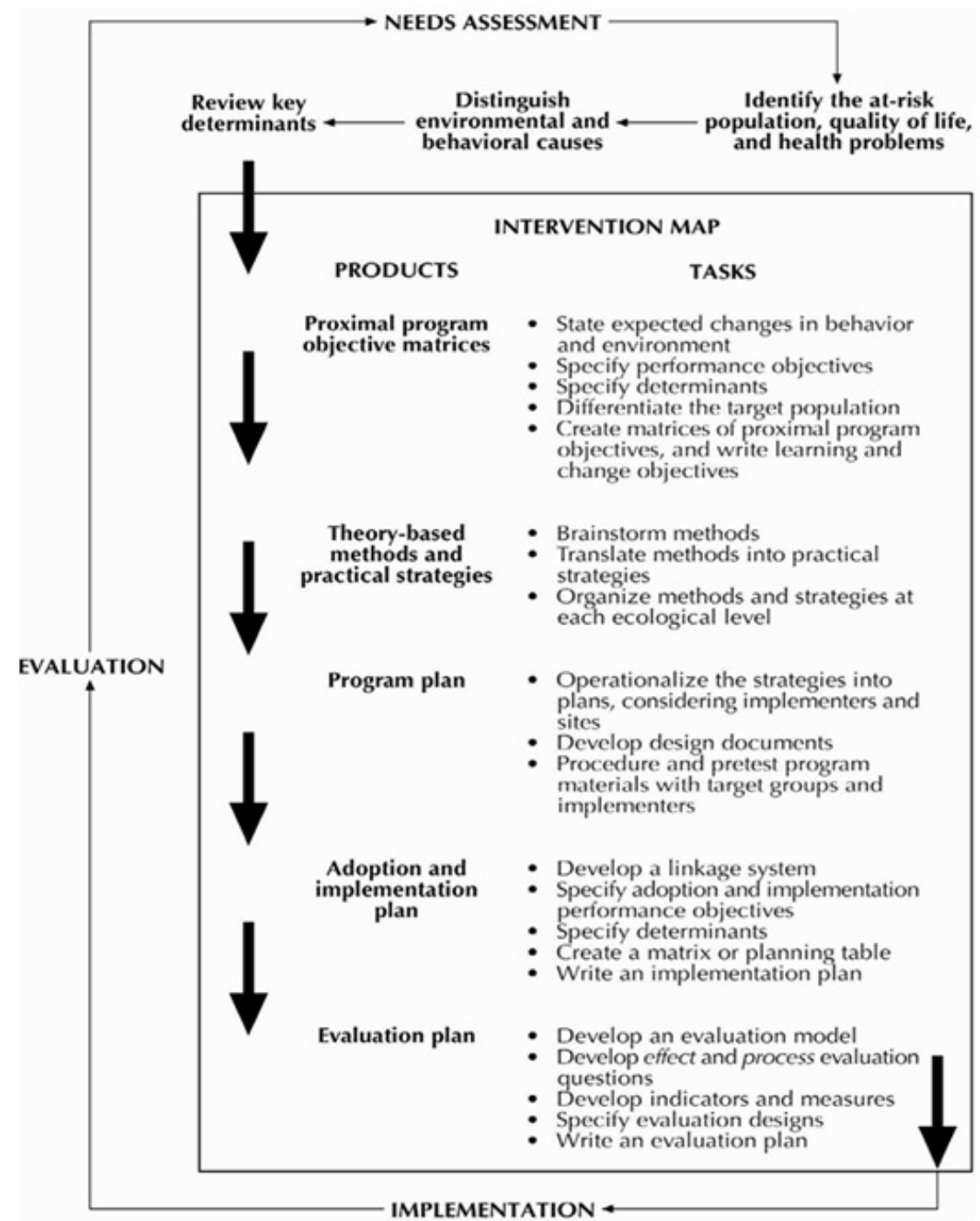

Abstract P274 Figure 1

methods and practical strategies, intervention design, adoption and implementation, and evaluation.

Findings The six stages demonstrated that self-management behaviours are a critical component of asthma care and that childhood asthma care may be influenced through behaviour and environment. The process showed how intervention methods based on self-regulatory theory (Leventhal et al., 1984) are applicable to self-management behaviours and can be translated into practical applications for asthma self-management.

Figure 1. Intervention mapping process diagram (Bartholomew et al., 2001)

Step one involved conducting a literature review, collecting preliminary data and developing the asthma PRECEDE model. Step 2 highlighted the at-risk group and explores relevant theories/frameworks e.g. Asthma self-management behavioural framework (Bartholomew et al., 2001). Performance objectives and determinants were established in order to devise a change objective matrix. Step 3 linked $\mathrm{BC}$ techniques to determinants and change objectives in order to change behaviour. Step 4 was the intervention design targeting asthma self-management. Key features were child centred teaching including a video and facilitating family/GP links. Step 5 encompassed the logistics of the intervention i.e. mode of delivery, costing and outcome expectations e.g. perceived benefits and better health. Step 6 outlined how the intervention would be evaluated including baseline and follow-ups, review of ED attendances, self-reported measures, Asthma Quality of Life Scale and Paediatric Asthma Control Test.

Discussion The intervention mapping process aided the design of an intervention tailored to a child's own data and to the specific needs of a child/family. The intervention should help a child progress to more advanced asthma management and promote a tie between child/family and GP. The next step is to implement and evaluate this intervention at Birmingham Children's Hospital to tackle the paediatric high rates of asthma hospital admissions.

Words: 345 (excluding subheadings and diagram)

\section{P275 PREVALENCE AND TREATMENT OF ACTIVE ASTHMA IN SCOTLAND USING THE PRESCRIBING INFORMATION SYSTEM}

doi:10.1136/thoraxjnl-2012-202678.367

${ }^{1} \mathrm{MFC}$ Steiner, ${ }^{1} \mathrm{G}$ Devereux, 'SW Turner, ${ }^{1} \mathrm{~J}$ McLay, ${ }^{2} \mathrm{~B}$ Bishop, ${ }^{2} \mathrm{G}$ Wyper. ${ }^{1}$ University of Aberdeen, Aberdeen, UK; ${ }^{2}$ NHS National Services Scotland, Edinburgh, UK

Background Many studies of the clinical epidemiology of asthma that have used routinely collected drug prescription or dispensing data have been limited to samples assumed to be representative of the national population from which they are drawn. Our aim was to describe asthma prevalence and treatment in children and young 
adults using the Prescribing Information System (PIS), a national prescribing and dispensing database for Scotland.

Methods For more than $95 \%$ of the dispensed prescriptions in primary care between December 2009 and November 2011 a valid patient identifier was available and the database includes some socio-demographical characteristics (age-group, sex, SIMD) of the patients. Data were also linked to hospital admission data. The analysis was limited to patients aged up to 44 years to reduce contamination by COPD.

Data

We identified 358,804 patients with 2,809,563 dispensed prescriptions for inhaled therapies used for asthma; equating to a prevalence of $11.4 \%$ of the 3,139,356 people aged 0-44 registered with a GP in Scotland. The age specific prevalence rates are detailed in the table. However, 95,207 patients had only one or two dispensed prescriptions for short-acting beta2-agonists (SABA) and no other inhaled therapies in the two years; we consider these patients to be unlikely to have active asthma (table). Additionally, 1,041 cases on inhaled therapy had hospital admission(s) with a diagnosis of COPD (ICD10: J40-J44) and are excluded from further analysis. $6,056(2.3 \%)$ of people collecting inhaled therapy ( $>2$ SABA) had at least one hospital admission with a primary diagnosis of asthma.

$18.4 \%$ of patients collected SABA only, $46.8 \%$ collected SABA + inhaled corticosteroids (ICS), $0.1 \%$ (371) collected SABA + long acting beta2 agonist (LABA) only, $13.6 \%$ collected SABA + combined ICS/LABA preparation, $2.1 \%$ collected SABA + ICS +LABA, 1.7\% collected ICS/LABA only, leukotriene receptor antagonists (LTRA) were collected by $8.1 \%$ and long acting antimuscarinic agents (LAMA) were collected by $1.0 \%$.

Conclusion This current and whole population database indicates that the prevalence of asthma is approximately $10 \%$ in young adults and $15 \%$ in children living in Scotland but prevalence of active asthma is approximately $8 \%$ in adults and $10 \%$ in children.

Abstract P275 Table 1 Dispensed inhaled therapy and hospital admission data from the Scottish NHS databases

\begin{tabular}{lccccc}
\hline $\begin{array}{l}\text { Age } \\
\text { groups }\end{array}$ & Population & $\begin{array}{c}\text { Inhaled } \\
\text { therapy(n) }\end{array}$ & Prevalence \% & $\begin{array}{c}\text { >2 SABA and/or } \\
\text { other (n) }\end{array}$ & $\begin{array}{c}\text { Prevalence of } \\
\text { active asthma }\end{array}$ \\
\hline $0-4$ & 289,464 & 45,223 & 15.6 & 25,041 & $8.7 \%$ \\
$5-14$ & 572,289 & 76,018 & 13.3 & 56,223 & $9.8 \%$ \\
$15-24$ & 713,676 & 73,446 & 10.3 & 53,985 & $7.6 \%$ \\
$25-44$ & $1,563,926$ & 164,117 & 10.5 & 128,348 & $8.2 \%$ \\
Total & $3,139,356$ & 358,804 & 11.4 & 263,597 & $8.4 \%$ \\
\hline
\end{tabular}

\section{P276 THE IMPACT OF THE OPTIMUM PATIENT CARE SERVICE ON OUTCOMES FOR ADULT ASTHMATIC PATIENTS}

\section{doi:10.1136/thoraxjnl-2012-202678.368}

${ }^{1} \mathrm{D}$ Ryan, ${ }^{2} \mathrm{~F}$ Ryan, ${ }^{2} \mathrm{~L}$ Mascarenhas, ${ }^{3} \mathrm{D}$ Saralaya, ${ }^{4} \mathrm{M}$ Britton, ${ }^{2} \mathrm{~J}$ von Ziegenweidt, ${ }^{2} \mathrm{~A}$ Burden, ${ }^{2} \mathrm{C}$ Hutton, ${ }^{2} \mathrm{~S}$ Gould, ${ }^{5 P}$ David. 'Woodbrook Medical Centre, Loughborough, United Kingdom; ${ }^{2}$ Research in Real Life, Cambridge, United Kingdom; ${ }^{3}$ Bradford Teaching Hospital, Bradford, United Kingdom; '4St Peter's Hospital, Chertsey, United Kingdom; ${ }^{5}$ University of Aberdeen, Aberdeen, United Kingdom

Introduction and Objectives The Optimum Patient Care (OPC) service provides a comprehensive asthma assessment, analysing both GP-recorded and patient-reported outcomes to generate patient-specific management recommendations (based on British guidelines) for considerations by practise staff. This study evaluates the effect of the OPC asthma service evaluation on real-life asthma control outcomes in a UK primary care adult asthma population compared with a control population.

Methods Routine and patient-reported questionnaire data were collected for 2952 patients with clinician-diagnosed asthma and 1 year outcome data managed in practises across Surrey and Leicester County and Rutland Primary Care Trusts. The effect of the OPC service was evaluated by assessing change in markers of asthma control between initiation and re-assessment: exacerbation frequency (Read code defined acute exacerbations and number of courses of acute oral steroids in previous 12 months) and risk status (high risk: $\geq 2$ annual exacerbations). The number of OPC management suggestions implemented by each practise was also considered. Change in exacerbations over the 12 month period was compared with a control group of patients from UK practises who did not receive OPC management recommendations. Exacerbation rate ratios ( $R R$ ) for treatment group were produced (relative to control), adjusted for baseline confounders (95\%CI).

Results There were 2952 patients in the study from 22 practises. At time of re-assessment $10 \%$ of OPC management suggestions had been implemented and $22 \%$ of patients managed at British Thoracic Society (BTS) steps $4 / 5$ had improved their risk status. $17.1 \%$ of patients in the control group $(n=22,952)$ suffered from $\geq 1$ exacerbation during the baseline year, increasing to $17.3 \%$ of patients after 12 months. For patients receiving the OPC management review, $24.8 \%$ had $\geq 1$ exacerbation during baseline, decreasing to $15.3 \%$ following review.

Conclusions OPC's combined patient-reported and practise data assessment allowed thorough patient assessment and the generation of tailored management recommendations. Although only $10 \%$ of recommendations were implemented at 12 months, the OPC service appeared to have a beneficial effect on risk status and exacerbation rates.

Abstract P276 Table 1 Rate Ratios for treatment group exacerbations, compared to control of patients not receiving the OPC management review service.

\begin{tabular}{lcc}
\hline Exacerbations & Control & Management review service \\
\hline RR adjusted for age, baseline & 1.00 & 0.74 \\
exacerbations & & $(0.66,0.83)$ \\
RR adjusted for age, asthma & 1.00 & 0.76 \\
management stage & & $(0.68,0.85)$ \\
\hline
\end{tabular}

\section{P277 STATE OF THE UNION - AN ASSESSMENT OF CURRENT ADULT ASTHMATICS, THEIR DEMOGRAPHICS, TREATMENT AND OUTCOMES IN 210 PRACTISES ACROSS THE UK}

doi:10.1136/thoraxjnl-2012-202678.369

${ }^{1} \mathrm{D}$ Price, ${ }^{2} \mathrm{~F}$ Ryan, ${ }^{2 \mathrm{~L}}$ Mascarenhas, ${ }^{2} \mathrm{D}$ West, ${ }^{2} \mathrm{~S}$ Gould, ${ }^{2} \mathrm{C}$ Hutton, ${ }^{3} \mathrm{D}$ Ryan. ${ }^{1}$ University of Aberdeen, Aberdeen, United Kingdom; ${ }^{2}$ Research in Real Life, Cambridge, United Kingdom; ${ }^{3}$ Woodbrook Medical Centre, Loughborough, United Kingdom

Abstract P277 Table 1 Asthma control and number of exacerbations per patient by BTS asthma management step

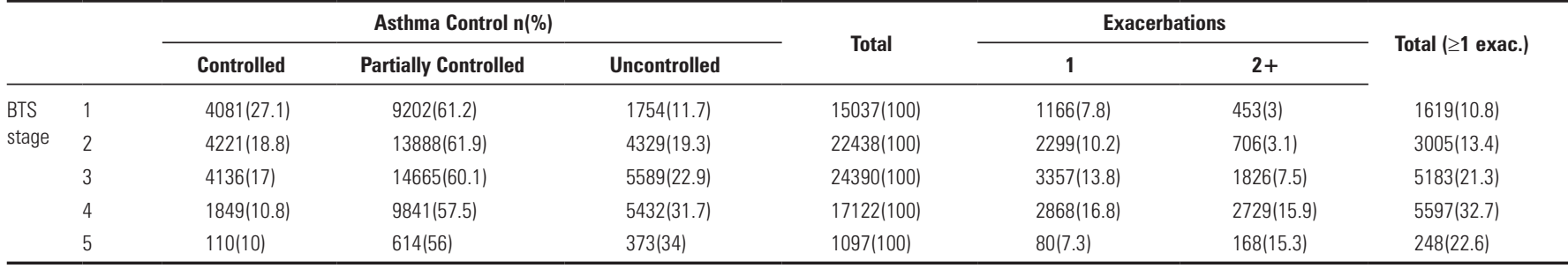

